\title{
Effects of the addition of coal tar pitch-based mesocarbon microbeads on the preparation of mesophase pitch
}

\author{
Xin Gong ${ }^{1}$, Xiaodong Liu ${ }^{1}$ and Dong Liu ${ }^{1, *}$ \\ ${ }^{1}$ State Key Laboratory of Heavy Oil Processing, China University of Petroleum, Qingdao, Shandong 266580, China
}

\begin{abstract}
Mesophase pitch was prepared by co-carbonization of the hydrocracking tail oil (HCTO) and coal tar pitch-based mesocarbon microbeads (MCMB). The effects of the addition amount of coal tar-based MCMBs on the $\mathrm{C} / \mathrm{H}$ atomic ratio, softening point, carbon residue, optical texture, aromatic lamellae stacking order degree, and chemical reaction kinetics of mesophase pitch were investigated. The results show that MCMB can be used as a nucleating agent to accelerate the nucleation of mesophase spheres and reduce the energy barriers for the formation of the anisotropic phase. However, excessive MCMB greatly increases the viscosity of the pitch matrix and weakens the mobility of condensed aromatic macromolecules, leading to the formation of mesophase pitch with a mosaic structure and poor lamellar orientation. When the addition amount of MCMB is $5 \mathrm{wt} \%$, the mesophase pitch with a large domain texture, high aromatic lamellar stacking order degree, and low softening point can be formed. This work provides a pathway to efficiently prepare mesophase pitch without catalyst removal.
\end{abstract}

\section{Introduction}

Mesophase pitch (MP) is composed of the oriented arrangement of planar polycondensation aromatic hydrocarbons, which can be used as a superior carbon precursor for the fabrication of advanced carbon materials, including carbon fiber, mesocarbon microbeads, and needle coke [1]. Generally, the petroleum-based raw material, such as FCC decant oil and hydrocracking tail oil (HCTO), contain abundant naphthenic structures and short alkyl side chains, which are regarded as a kind of premium precursor for preparation mesophase pitch. However, the preparation of petroleum-based mesophase pitch by thermal polycondensation need a longer reaction time, which increases the industrial cost greatly [2].

To further promote the formation rate of petroleumbased mesophase pitch and shorten the thermal polycondensation time, many researchers tried to accelerate the formation process of mesophase by adding various metallic compounds. Iron-containing compound, such as ferrocene and $\mathrm{FeCl}_{3}$, is a common type of catalyst which accelerates the formation of the mesophase pitch [3]. These iron-containing compounds can dissociate iron ions under the thermal polycondensation system. Then, iron ions can act as Lewis acid to catalyse polymerization reactions, leading to the growth and coalescence of mesophase spheres in a short time. Nevertheless, iron ions are easily deactivated due to the chemical reaction with sulphides existed in the petroleum pitch. Additionally, the effects of other metallic compounds, including $\mathrm{AlCl}_{3}, \mathrm{Al}_{2} \mathrm{O}_{3}, \mathrm{ZrCl}_{4}$, and $\mathrm{TiO}_{2}$ on the formation of mesophase pitch were also reported in the literature [4-6]. However, it is difficult to remove completely the metal catalysts from the pyrolytic products. The residual metal catalyst would decrease the graphitization degree of mesophase pitch. To avoid the problem of catalyst removal, many scholars considered that the mesophase pitch or isotropic pitch containing aromatic macromolecules can promote the formation of mesophase pitch. As nucleating agents, the isotropic pitch or mesophase pitch has good compatibility with the raw material pitch and without the problem of catalyst removal, which is a kind of ideal additive to accelerate the thermal polycondensation reaction [7-9].

In this work, mesophase pitch were prepared by cocarbonization of hydrocracking tail oil (HCTO) and coal tar pitch-based mesocarbon microbead (MCMB) to shorten the thermal polycondensation time and avoid the removal of catalysts. MCMB is a kind of spherical carbon material formed by the oriented alignment of planar polycondensation aromatic hydrocarbons, which is the primary structure of mesophase pitch. The effect of MCMB on the chemical composition, morphology, and microstructure of petroleum-based mesophase pitch was investigated.

\section{Experiment}

\subsection{Materials}

Hydrocracking tail oil (HCTO) was provided by CNOOC. The properties of HCTO are shown in Table 1. MCMB was homemade by thermal polymerization of coal tar pitch.

\footnotetext{
*Corresponding author: liudong@upc.edu.cn
} 
Table 1. The properties of HCTO

\begin{tabular}{|c|c|c|}
\hline \multicolumn{2}{|r|}{ Items } & HCTO \\
\hline \multicolumn{2}{|c|}{ Density $\left(20^{\circ} \mathrm{C}\right) / \mathrm{kg} \cdot \mathrm{m}^{-3}$} & 1100 \\
\hline \multicolumn{2}{|c|}{ Viscosity $\left(100^{\circ} \mathrm{C}\right) / \mathrm{mm}^{2} \cdot \mathrm{s}^{-1}$} & 26.32 \\
\hline \multirow{5}{*}{$\begin{array}{l}\text { Elementary } \\
\text { analysis }\end{array}$} & C/wt.\% & 87.93 \\
\hline & H/wt.\% & 9.94 \\
\hline & S/wt. \% & 0.44 \\
\hline & N/wt. $\%$ & 0.61 \\
\hline & $\mathrm{C} / \mathrm{H}^{\mathrm{a}}$ & 0.74 \\
\hline \multirow{3}{*}{ n-d-M method } & $\mathrm{C}_{\mathrm{A}} \mathrm{a} / \mathrm{wt} \%$ & 54.0 \\
\hline & $\mathrm{C}_{\mathrm{N}}^{\mathrm{b}} / \mathrm{wt} \%$ & 23.9 \\
\hline & $\mathrm{C}_{\mathrm{P}}^{\mathrm{c}} / \mathrm{wt} \%$ & 22.1 \\
\hline \multirow{4}{*}{$\begin{array}{c}\text { SARA } \\
\text { analysis }\end{array}$} & Saturates/wt.\% & 22.3 \\
\hline & Aromatics/wt. $\%$ & 63.0 \\
\hline & Resins/wt.\% & 13.7 \\
\hline & Asphaltenes/wt.\% & 0 \\
\hline
\end{tabular}

\subsection{Preparation of MP}

Various proportions of coal tar pitch-based MCMB (0 $\mathrm{wt} \%, 1 \mathrm{wt} \%, 3 \mathrm{wt} \%, 5 \mathrm{wt} \%, 7 \mathrm{wt} \%$ ) and HCTO were mixed uniformly by ultrasonication. The mixture was poured into an autoclave equipped with a stirring device and heated to $440{ }^{\circ} \mathrm{C}$ for $6 \mathrm{~h}$, during which the stirrer speed was maintained at $500 \mathrm{r} / \mathrm{min}$. The resultant pitches were cooled down, denoting as MP-0, MP-1, MP-3, MP5, and MP-7.

\subsection{Characteristics}

XP-4030 metallographic microscope was used to observe and obtain the optical imagines of mesophase pitch. X-ray diffraction (XRD) analysis of mesophase pitch was performed on an X'Pert Pro MPD model Xray diffractometer with $\mathrm{Cu} \mathrm{K} \alpha$ radiation. The softening point of mesophase pitch was measured using a ringand-ball apparatus. The carbon residue was measured according to the GB8727-88 standards. The Fourier Transform Infrared spectra (FTIR) was taken by NEXUS FT-IR spectrometer equipped with a mercury-cadmium. Its scanning range was from $400 \mathrm{~cm}^{-1}$ to $4000 \mathrm{~cm}^{-1}$ by averaging 32 scans. Three solvents, including n-heptane, toluene, and pyridine were employed to explore the solubility of mesophase pitch. The elemental analysis of the products was performed on a PE-2400 Series HCSN elemental analyser. The structural order degree of mesophase pitch also were carried out on the DXR Raman microscope using $514 \mathrm{~nm}$ laser excitation.

\section{Results and discussion}

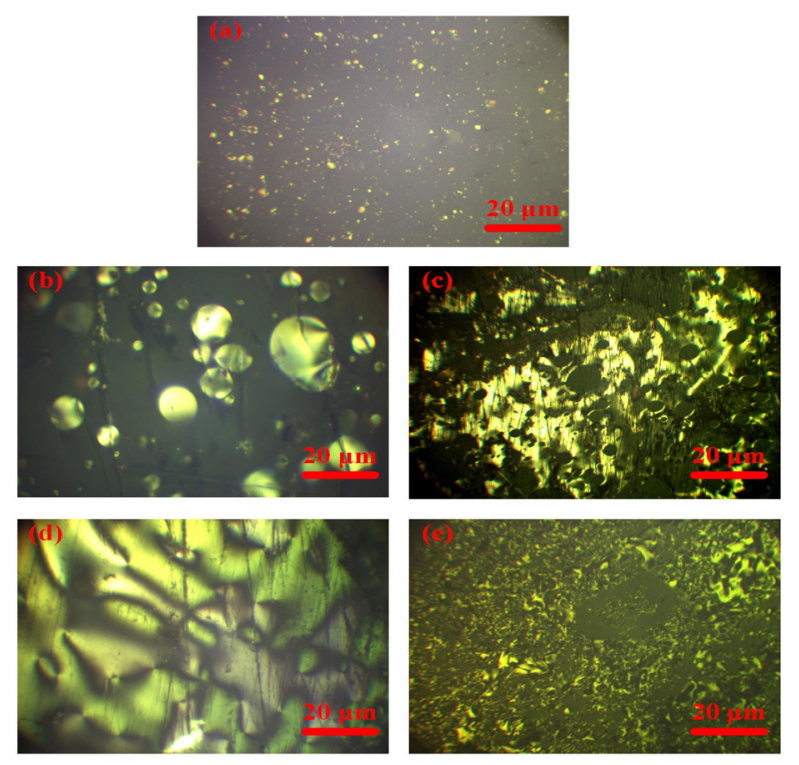

Figure 1. Optical photographs of MP with different amounts of MCMB: (a)MP-0; (b) $1 \mathrm{wt} \%$; (c) $3 \mathrm{wt} \%$; (d) $5 \mathrm{wt} \%$; (e) $7 \mathrm{wt} \%$

Figure 1 exhibits the optical polarized images of the mesophase pitch. The dark parts and bright parts represent the isotropic phase and anisotropic phase, respectively. When the additive is absent (Figure 1a), a number of mesophase spheres (the size $\leqslant 1 \mu \mathrm{m}$ ) and a few spheres aggregates can be observed. The anisotropic content of MP-0 is the lowest among the five pyrolytic products. As the additive amount of MCMB increased from $1 \mathrm{wt} \%$ to $5 \mathrm{wt} \%$ (seen in Figure $1 \mathrm{~b}-\mathrm{d}$ ), the anisotropic content of MP increases gradually, and the optical texture changes from large mesophase spheres (diameter $<10 \mu \mathrm{m}$ ) to a large domain (diameter $>100$ $\mu \mathrm{m})$. This indicates that the formation and transformation behaviours of the anisotropic phase are accelerated with the increase of the additive amounts of MCMBs. The reason is that the MCMB can be used as the nucleation agent to promote the rate of the growth and coalescence of mesophase spheres. Significantly, mesophase pitch with mosaic texture was formed when the MCMB content is $7 \mathrm{wt} \%$ (as seen in Figure 1e) which is because excessive MCMBs increased the viscosity of pitch matrix, leading to suppression of the growth and coalescence of mesophase spheres. 


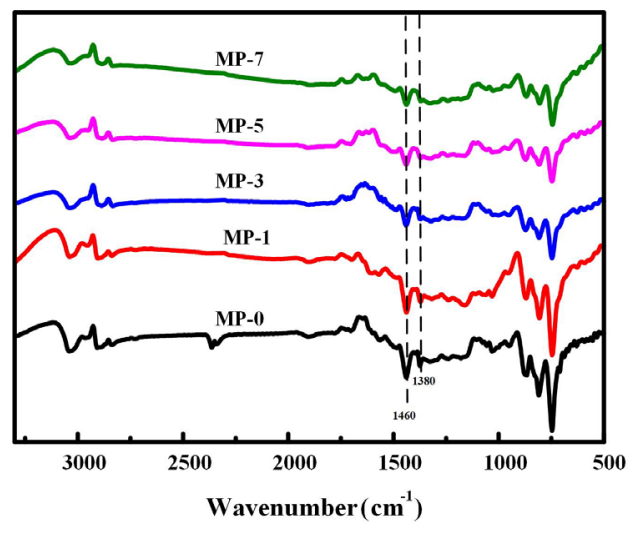

Figure 2. FTIR spectra of the pyrolyzed pitch

The FTIR spectra of mesophase pitch doped with different amounts of MCMBs are shown in Figure 2. Obviously, five kinds of mesophase pitch all exhibit the bending vibration absorption peaks of $-\mathrm{CH}_{3}$ (near 1380 $\mathrm{cm}^{-1}$ ) and $-\mathrm{CH}_{2-}$ (around $1460 \mathrm{~cm}^{-1}$ ). It can be seen that the aliphatic $\mathrm{C}-\mathrm{H}$ bonds gradually weakens with the increase of MCMBs, indicating that the addition of the MCMBs can promote the cracking of alkyl side chains and the dehydrogenation condensation reaction. Besides, the out of plane bending vibration peaks of polynuclear polycyclic aromatic hydrocarbons (PAHs) appear between $878 \mathrm{~cm}^{-1}$ and $618 \mathrm{~cm}^{-1}$ for all five kinds of asphalts. The ortho substitution index $\left(I_{o s}\right)$ can be calculated according to the following formula [10] :

$$
I_{o \mathrm{~s}}=A_{750} /\left(A_{750}+A_{814}+A_{840}+A_{880}\right)
$$

In the formula, $\mathrm{A}_{750}, \mathrm{~A}_{814}, \mathrm{~A}_{840}$, and $\mathrm{A}_{880}$ represent absorption intensities at $750,814,840$, and $880 \mathrm{~cm}^{-1}$, respectively. The value of $I_{o s}$ can reflect the condensation degree of polycyclic aromatic hydrocarbons. The $I_{o s}$ values of five kinds of mesophase pitch are 0.380 (MP-0), 0.356 (MP-1), 0.340 (MP-3), 0.319 (MP-5), and 0.303 (MP-7), respectively, implying that the condensation degree of mesophase pitch increases with the addition of MCMBs. Combined with the results of FTIR analysis and optical images of mesophase pitch, it can be deduced that MCMBs can not only act as a "nucleating agent" to accelerate the nucleation of mesophase spheres and promote the coalscence but also enhance the dehydro condensation of small aromatic molecules in the feedstock to form condensed aromatic macromolecules with high molecular weight.

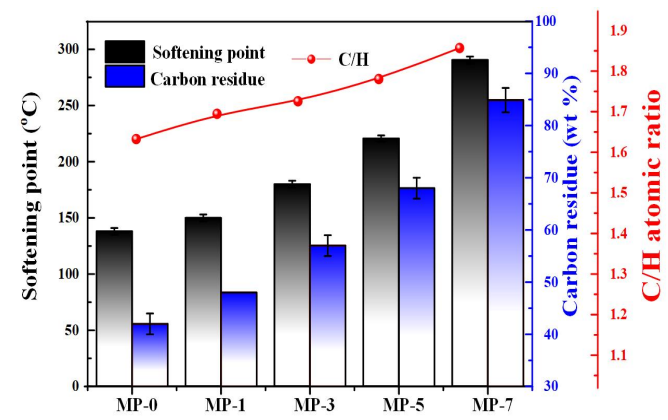

Figure 3. The variation trends of softening point, carbon residue, and $\mathrm{C} / \mathrm{H}$ atomic ratio of pyrolysis products.
The variation trends of softening point, carbon residue, and $\mathrm{C} / \mathrm{H}$ atomic ratio of mesophase pitch doped with different amounts of MCMB are shown in Figure 3. As the amount of MCMB increases from $0 \mathrm{wt} \%$ to 5 $\mathrm{wt} \%$, the softening point continually increases from $138{ }^{\circ} \mathrm{C}$ to $223{ }^{\circ} \mathrm{C}$, and the carbon residue increases from $42 \mathrm{wt} \%$ to $68 \mathrm{wt} \%$. This indicates that the presence of the MCMB accelerates the formation of condensed aromatic macromolecules by the nucleation process. Significantly, when the additive amount of MCMB increases to $7 \mathrm{wt} \%$, the softening point and carbon residue increase sharply. The softening point and carbon residue of MP-7 are $290{ }^{\circ} \mathrm{C}$ and $84 \mathrm{wt} \%$, respectively, which are much higher than those of other samples. The reason is that excessive MCMB can increase the viscosity of the pitch matrix dramatically and restrict the hydrogen transfer reaction in the system, leading to the disordered cross-linked reaction of aromatic macromolecule free radicals. The results contribute to the formation of condensed aromatic macromolecules with poor flatness, resulting in the mosaic texture of mesophase pitch. Besides, the change tendency of the $\mathrm{C} / \mathrm{H}$ atomic ratio is also exhibited in Figure.3. With the increase of $\mathrm{MCMBs}$, the $\mathrm{C} / \mathrm{H}$ atomic ratio shows an increasing trend, confirming that doping MCMBs into the carbonization system can increase the condensation degree of mesophase pitch.

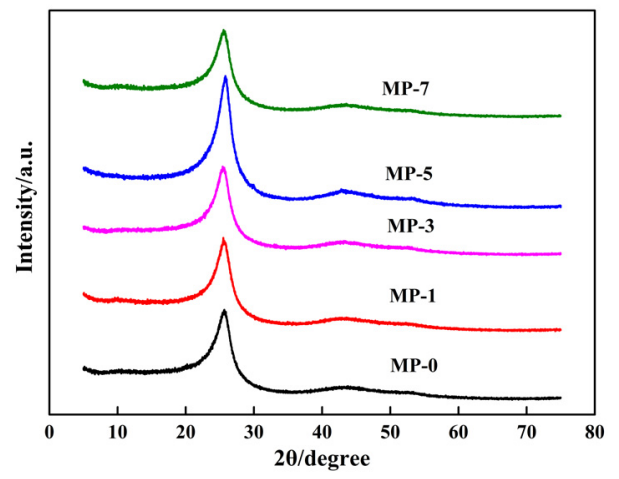

Figure 4. X-ray diffraction patterns of mesophase pitch doped with various contens of MCMB

Table 2. The microstructure parameters of pyrolysis products

\begin{tabular}{ccccc}
\hline Sample & $\mathrm{d}_{002} / \mathrm{nm}$ & $\mathrm{L}_{\mathrm{c}} / \mathrm{nm}$ & $\mathrm{n}$ & $\mathrm{I}_{\mathrm{D}} / \mathrm{I}_{\mathrm{G}}$ \\
\hline MP-0 & 0.350 & 3.76 & 11.7 & 0.970 \\
MP-1 & 0.349 & 3.80 & 11.9 & 0.933 \\
MP-3 & 0.347 & 3.86 & 12.1 & 0.888 \\
MP-5 & 0.345 & 4.30 & 13.4 & 0.702 \\
MP-7 & 0.348 & 3.93 & 12.3 & 0.920 \\
\hline
\end{tabular}

Figure 4 shows the XRD patterns of mesophase pitch doped with different contents of MCMB. The interlayer spacing $\left(d_{002}\right)$, stacking height $\left(L_{\mathrm{C}}\right)$, and stacking layer number (n) calculated according to the Bragg-Scherrer formula. The results are listed in Table 2. It can be seen from Figure 7 that all the mesophase pitch possess obvious 002 diffraction peaks (near 26\%). When the addition amount continually increases to $5 \mathrm{w} \%$, the interlayer spacing $\left(d_{002}\right)$ shows a decreasing trend, 
whereas the corresponding stacking height $\left(L_{\mathrm{C}}\right)$ exhibits an increasing trend (seen in Table 2), demonstrating that the moderate addition amount of MCMB can improve the stacking order degree of aromatic lamellae of mesophase pitch. However, when the addition amount of MCMB is $7 \mathrm{wt} \%$, the increase in interlayer spacing $\left(d_{002}\right)$ and the decrease in stacking height $\left(L_{\mathrm{C}}\right)$ lead to the reduction of stacking layer number (n) from 13.4 (MP-5) to 12.3 (MP-7). This result confirmed that excessive MCMBs decrease the stacking order degree of aromatic lamellae in mesophase pitch, mainly due to the formation of aromatic macromolecules with poor planar by the excessive condensation in the carbonization system. The analysis result of XRD is in agreement with the phenomenon showed by polarized micrographs.

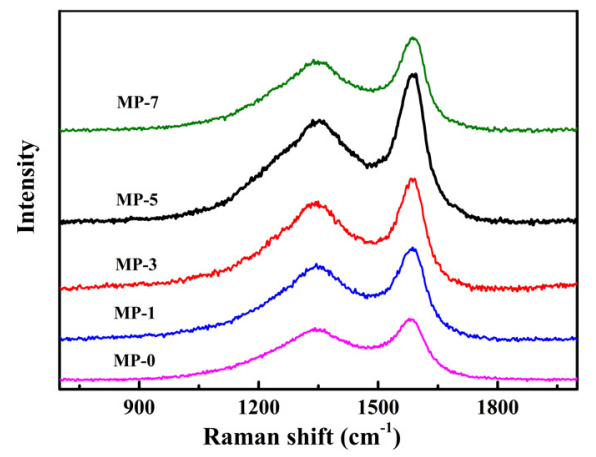

Figure 5. Raman spectra of the pyrolysis products

The Raman spectra of mesophase pitch doped with different contents of MCMBs are shown in Figure 5. Two obvious peaks around $1360 \mathrm{~cm}^{-1}$ and $1580 \mathrm{~cm}^{-1}$ are found for each sample, corresponding to the D band and $\mathrm{G}$ band, respectively. The former and latter represent the disordered graphite lattice ( $\mathrm{D}$ band) and the ideal graphite lattice ( $G$ band), respectively. The intensity ratio $\left(\mathrm{I}_{\mathrm{D}} / \mathrm{I}_{\mathrm{G}}\right)$ is used to definitely reflect the microstructural order degree of carbon materials, and the values of $\mathrm{I}_{\mathrm{D}} / \mathrm{I}_{\mathrm{G}}$ are listed in Table 2 . The $\mathrm{I}_{\mathrm{D}} / \mathrm{I}_{\mathrm{G}}$ ratio of mesophase pitch exhibits a tendency of first decreasing and then increasing, indicating that the structural order degree increases first and then decreases with the growth of MCMBs contents. Significantly, MP-5 possesses the lowest values of $I_{D} / I_{G}(0.702)$ among the five samples, implying that MP-5 exhibits the highest structural order degree and the least structural defects. These analysis results are consistent with the result of XRD analysis.

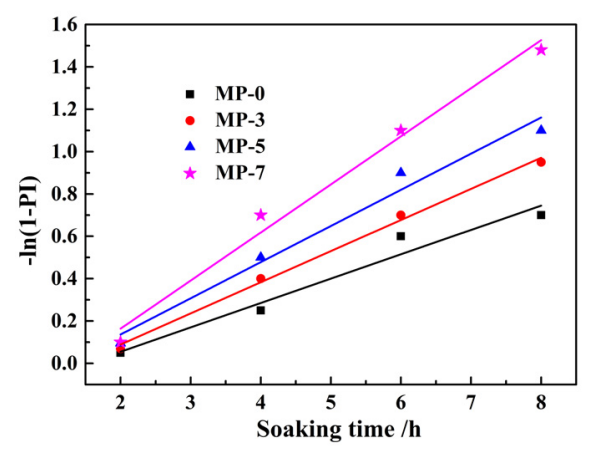

Figure 6. Functional relation between PI content of mesophase pitch and soaking time
To further explore the effect of MCMB content on the chemical kinetics of mesophase pitch formation, the functional relation between PI content and soaking time was established, shown in Figure 6. The chemical kinetic model was achieved based on the following two assumptions [11]: (1) PI content of mesophase pitch represents the anisotropic content in the pyrolysis product; (2) the entire process of mesophase pitch formation obeys the first-order kinetics. According to the above equation, there is a linear relationship between $\ln (1-\mathrm{PI})$ and the reaction time. The calculated reaction rate constants of MP-0, MP-3, MP-5 and MP-7 are 0.11, $0.15,0.17$, and 0.23 , respectively. It can be found that the carbonized rate of the feedstock gradually increases with the increase of the additive amount of MCMB. The result of chemical kinetic analysis reveals that MCMB can significantly promote the polymerization and condensation reaction rate. However, when the addition amount is $7 \mathrm{wt} \%$, the reaction constant of MP-7 is much higher than those of other pyrolysis products. The most reasonable explanation is that excessive MCMB causing a rapid increase in the viscosity of the reaction medium can restrict the hydrogen transfer reaction, leading to the excessive condensation of the pitch matrix. Therefore, the appropriate amount of MCMB can avoid excessive condensation and promote the formation of mesophase pitch with wide-area streamline texture.

\section{Conclusion}

In this paper, the effect of the addition of coal tar pitchbased MCMB on the preparation of mesophase pitch is investigated. The MCMB can significantly promote the polymerization and condensation reactions of aromatics and accelerate the formation of mesophase spheres by the nucleation process. However, excessive MCMBs will greatly increase the viscosity of the pitch matrix and hinder the hydrogen transfer reaction, leading to the formation of mesophase pitch with mosaic texture and poor stacking order degree of aromatic lamellae. Therefore, the co-carbonization of MCMB with an appropriate amount $(5 \mathrm{wt} \%)$ and HCTO can obtain the mesophase pitch with a high structural order degree and large domain texture. This work is of great significance for the preparation of high-quality mesophase pitch.

\section{References}

1. D. Liu, R. Yu, J.P. Niu, B. Lou, Y.E. Fu, S.H. Guo, N. Zhao, E.Q. Yu, Fuel 270, 17529 (2020)

2. J.G. Kim, J.H. Kim, J.S. Im, Y.S. Lee, T.S. Bae, J. Ind. Eng. Chem 62, 176-84 (2018)

3. M. Braun, J. Kramer, K.J Hüttinger, Carbon 29, 439-448 (1995)

4. J.F. Boero, J.A. Wargon, Carbon 19, 333-340 (1981)

5. S. Kumar, M. Srivastava, J. Anal. Appl. Pyrolysis 140, 192-200 (2015)

6. T.Q. Li, C.Y. Wang, J.M. Zheng, H.Wang, Fuel. Process. Technol 87, 77-83 (2005) 
7. X.W. Zhang, Y.C. Meng, B.L. Fan, Z.K. Ma, H.H. Song, Fuel 243, 390-397 (2019)

8. S.H. Lee, S.M. Lee, U. Im, S.D. Kim, S.H. Yoon, B.R. Lee, D.H. Peck, Y.G. Shul, D.H. Jung, Carbon 155, 186-194 (2019)

9. L. Lei, X.C. Lin, J. He, Y.K. Zhang, J.X. Lv, Y.G. Wang, J. Anal. Appl. Pyrolysis 155, 105039 (2021)
10. R. Yu, D. Liu, B. Lou, W. Zhu, S.H. Guo, X. Gong, J.S. Ye, C.Zhu, Fuel 247, 97-107 (2019)

11. X.W. Zhang, Z.K. Ma, Y.C. Meng, M. Xiao, B.L. Fan, H.H. Song, Y.Z. Yin, J. Anal. Appl. Pyrolysis 140 , 274-280

(2019) 